\title{
Consciousness \& Time: A Time-Based Model of the Evolution of Consciousness
}

\author{
Brad Bowins \\ Centre for Theoretical Research in Psychiatry \& Clinical Psychology, Toronto, Canada \\ Email: brad.bowins@bellnet.ca
}

How to cite this paper: Bowins, B. (2017) Consciousness \& Time: A Time-Based Model of the Evolution of Consciousness. Journal of Behavioral and Brain Science, 7 , 9-20.

http://dx.doi.org/10.4236/jbbs.2017.71002

Received: December 5, 2016

Accepted: January 17, 2017

Published: January 20, 2017

Copyright (c) 2017 by author and Scientific Research Publishing Inc. This work is licensed under the Creative Commons Attribution International License (CC BY 4.0).

http://creativecommons.org/licenses/by/4.0/

(c) (i) Open Access

\begin{abstract}
A novel theoretical model is presented maintaining that consciousness evolved on the basis of time distinctions. Various models of time pertain to the existence of future, present and past. It is proposed that the future represents potentialities, the present the actualization of certain potentialities, and the past a record of actualized potentialities. Actualization of potentialities derives from micro quantum wave function collapses with specific constellations corresponding to macro level form. Consciousness provides for an awareness of potentialities being actualized in the present, the time frame of consciousness closely aligning with the time frame of potentialities being actualized in the moment. Evolution of such awareness is highly probable, given the ensuing motivation enabling behavior to be altered in the moment to minimize the actualization of maladaptive potentialities, and maximize the actualization of adaptive potentialities. The model also provides a logical proof for the occurrence of time distinctions.
\end{abstract}

\section{Keywords}

Consciousness, Evolution of Consciousness, Psychological Arrow of Time, Time, Space-Time, Quantum

\section{Introduction}

Although seemingly unrelated at first glance, consciousness and time appear to be linked. But the nature of this linkage is unclear [1] [2]. At the heart of the mystery is confusion regarding what time actually represents, and whether our perception of time passing from future to present to past-the psychological arrow of time-is just an illusion or meaningful. Most directly addressing this matter is the work of Linde [1] and Smythies [2]. Linde [1] postulates that the universe consists of space-time, consciousness, and matter. Building on the work of Linde [1], Smythies [2] proposes that consciousness interacts with a static 
space-time universe where there is no clear separation of future, present and past, to provide us with our perception of time.

A problem with Smythies [2] perspective is that if consciousness is part of an unchanging universe that just exists, how can we discern time distinctions? Or in other words, if consciousness is unchanging, it should be impossible for it to discern change. Smythies [2] attempts to solve this problem by postulating a phenomenal consciousness space independent from physical reality, based on data demonstrating that in contrast to direct realism, our conscious experience is not a replica of reality but an interpretation. This conscious phenomenal space can then freely interact with space-time creating the perception of time distinctions when none exist [2]. Imagine this hypothesized conscious phenomenal space perceiving different related slices of space-time as time 1, time 2 and time 3 , or future, present and past.

While potentially feasible, a major problem exists with Smythies's [2] interpretation, namely that it represents a form of mind-body dualism: the conscious mind must somehow be distinct from the body in order to freely interact with static space-time. Research has demonstrated that we are intuitive dualists, separation being a default strategy [3] [4]. Various thought experiments were employed by Forstmann \& Burgmer [3] to assess how we cognitively process the mind and body. Physical properties were consistently seen as being retained compared to mental properties, and under conditions that taxed cognitive resources or primed them with an intuitive thinking style, mind-body dualistic beliefs were intensified [3]. The authors indicate that despite scientific advances in understanding the neurological origins of mental life, we are still essentially mind-body dualists. Anglin [4] found that subjects tended to locate the self and mind in the head, whereas the soul or essence of a person was in the chest, reflecting our propensity to see discreteness in entities like mind, body and soul. In contrast to perceptions of mind-body dualism, there is actually synchrony between the mind and body [5] [6].

A fundamental flaw that is at the heart of much of the mind-body dualistic approach is not seeing that the mind and components such as consciousness are body too! The conscious mind is brain, and not some free-floating entity, and the brain is part of the body. The linkage of the central and peripheral nervous systems and also neurotransmitters within and outside of the brain in diverse tissues, demonstrate this connection [5]. Assuming that consciousness cannot be separated as a phenomenal space from brain functioning, then consciousness should not be able to perceive time distinctions in a static space-time universe. Consciousness should be embedded in the fabric of the universe with the brain it is part of, only perceiving that slice of space-time, and not further distinctions. However, we do perceive time distinctions consisting of future, present and past, although the exact way of framing them can vary between cultures and even circumstances [7]. In a static space-time with mind and body inseparable, this cannot occur, necessitating another way of viewing space-time and its linkage with consciousness. 
I propose a unique model of space-time and conscious, maintaining that the psychological arrow of time actually aligns with the nature of time itself. For the most part physics devalues the role of life and intelligence in the universe, despite that very intelligence providing the understanding that makes physics a viable discipline. In opposition to this perspective, it seems reasonable that the psychological arrow might shed light on the nature of time itself, and also the linkage between time and consciousness. I postulate that consciousness evolved on the basis of actual time distinction. Before presenting my theory a summary of consciousness and time is required.

\section{Consciousness}

Consciousness entails many elements but what distinguishes it from unconsciousness is awareness, and as a prelude arousal [8] [9] [10]. Attention is commonly believed to distinguish consciousness but unconscious attention occurs, as revealed by the interesting phenomenon of blind sight, whereby those with damage to the occipital cortex, who are blind in any conscious sense, are able to navigate around a room full of objects without conscious attention [11] [12]. At some unconscious level rudimentary visual sensory images likely bypassing the occipital cortex are attended to, and appropriate responses are executed [11] [12]. Hence, attention cannot be used to distinguish consciousness and unconsciousness. Awareness, though, does seem to be restricted to consciousness, and we are unaware of unconscious states [8].

Another common misconception of consciousness is that only humans have the capacity. According to Fabbro et al. [9], there are 5 lines of evidence supporting the presence of consciousness in a wide range of animal species: First, a pattern of EEG activity in the range of $20-70 \mathrm{~Hz}$, typically linked to wakefulness and REM sleep; second, thalamo-cortical activity; third, widespread brain activity during processing of sensory stimuli; fourth, selective synchronization at cortical and brainstem levels of dynamically formed neural networks involved in binding sensory stimuli; fifth, the presence of egocentric maps for localizing an individual in a given space. Fish, amphibians, reptiles, birds, mammals and primates, demonstrate some form of consciousness based on these parameters [9] [13]. Indeed, it seems that once a minimal level of cognitive sophistication is achieved an organism becomes conscious.

Given the energy involved in consciousness and its evolution in such a wide range of animal species, there is likely to be an evolutionary adaptive function. Very energy intensive capacities fade from the gene pool if not adaptive, and certainly do not evolve in such diverse species over millions of years once a minimum level of cognitive sophistication is achieved [14]. It might be opinioned that consciousness could have arisen in an early vertebrate species and simply continued on in others. However, if not adaptive in these later species the trait would likely have disappeared from the gene pool. Also, beyond vertebrates, it appears that consciousness occurs in more cognitively sophisticated invertebrates, such as cephalopods including octopus, squid and cuttlefish [15] [16], 
and possibly also anthropods [17]. A more general critique is that this perspective represents adaptationism - a trait exists, therefore, it is useful, and so must have evolved. In this regard, a high bar of acceptance must be applied, and with consciousness there does appear to be a very distinct and powerful evolutionary fitness value related to time, that will be presented.

\section{Time}

The nature of time has been endlessly debated and there are different aspects that can be focused on. As an important note, as described by Einstein [18] under special relativity, time does not exist in isolation from space, with both linked as space-time. When time is discussed in this paper it is with the understanding that it is not isolated from space. Of relevance here is the relationship between future, present and past, and the psychological arrow of time, whereby we view time as passing from future to present to past. Four models of time describing the relationship between future, present, and past consist of:

- 4-D Block Model (the 4 referring to 3 spatial and 1-time dimension) maintaining that past, present and future are not absolutely distinguishable as part of the space-time landscape. Hence, there is no actual passage from future to present to past; they just occur. In a diagram form, they appear as an elongated rectangle without clear borders [19] [20]. This model applies to Smythies [2] perspective reviewed earlier.

- Moving Spotlight Model also predicting that past, present and future are part of the space-time landscape, but suggests that there is there is a privileged advancing present, that can be represented by a narrow bar on the rectangle [19] [20].

- Growing Block Model holding that there is a past arising from a present, but no future. In diagrammatic form, the forward section of the rectangle representing the future is missing [19] [20].

- Presentism sharply contrasts with the 4-D Block Model because it argues that only a narrow present occurs, the past and future not being real [19] [20].

The author's model of time with future, present and past distinctions, aligning with the psychological arrow of time, will now be presented. Our perception of time in terms of future-present-past (the psychological arrow of time) has been discredited as an illusion [2] [21]. In contrast to this notion, our perception of time passing from future to present to past is arguably our most stable perception. Consider a different way of viewing the past and future. Take the statement, "Yesterday I'm going to fly to Florida." What is your reaction? Insane, might be your immediate response, but even psychotic individuals would likely react in this way. How about, "Tomorrow I went to Florida, and the flight was fast." Uniformly people would respond that this is impossible, because it is.

Options for "time travel" outside of science fiction scenarios are very limited consistent with the notion of distinctions between future, present, and past. Time dilation allows a person to travel to another person's future, so long as the time traveler is separated in space by leaving the planet and shortly the solar 
system, moving close to the speed of light [18]. When the time traveler returns to Earth he or she has hardly aged, but the other person has aged much more; the time traveler has arrived in the person's future. However, even time dilation does not allow the time traveler to venture to his or her own past or future, or the other person's life prior to when they separated [22] [23] [24] [25]. Other options for actual time travel, such as space-time fully warping around on itself, such that you return to your own past, seem to be impossible, or at least there is no evidence that this is viable [26]. Hence, the universe appears to be structured such that interconnected and entangled entities must abide by approximately the same future, present and past. If the entities become disentangled in space (space-time) then time dilation is viable, but this is limited to returning to another person's future after separating.

If entangled entities must abide by future, present and past, how might this align with scientific knowledge? To generate this understanding we must look at what future, present and past represent. Regarding the future, it appears to consist of potentialities varying in probability. Consider what you are doing at the moment, and what you might be doing in fifteen minutes. There are many potential options, with probability values. For example, if you're reading, then the probability of reading and further along in the paper is greater than sleeping. It is said in physics that anything that is not impossible (according to the laws of physics and the universe) is possible, and hence has some non-zero probability. This perspective on the future is much different than the common notion of a set future that we can travel to.

In terms of the present time frame, certain future potentialities are actualized based on matter-energy interactions. For example, if in fifteen minutes you are reading and further along in the paper, then that potentiality is actualized. If you are tired and/or bored falling asleep, then that potentiality is realized. This occurrence aligns with how we can never undo what transpires in the very brief present moment. If you shout an obscenity at your partner or boss, it cannot be reversed. Likewise, if while too absorbed in your mobile device you step out in the path of a car, the collision and injuries are real. Frequently, we wish that negative occurrences can be reversed, but this is not possible. The time frame for actualizing future potentialities appears to be in the millisecond to at most a few seconds range. Actualized potential occurrences represent the past. We all have a past based on what has occurred, and this past cannot be erased or altered.

Potentialities varying in probability (future), actualization of certain potentialities (present), and actualized potentialities (past) aligns with the psychological arrow of time, although there is no "flow" per se. This explanation for the psychological arrow of time is consistent with the quantum physics principle of collapse of the wave function [27] [28]. Potential states of a quantum system are represented by wave functions possibly as a superposition [27] [28]. When a measurement occurs detecting one of these states the wave function for that one stands out, a process described as collapse of the wave function [27] [28]. The measurement represents a matter-energy based interaction with the given state, 
and hence an actual measurement is likely not required [29] [30]. There are different ways that collapse of the wave function might occur, such as that for a given potential state standing out while all others vanish, all potential states funneling into the actualized one, or all continuing but only one being distinguished in some fashion [27] [28].

At this point critics will point out that collapse of the wave function is a quantum micro process, and simply cannot occur at a macro level, an entirely accurate statement. However, macro form is derived from micro and largely quantum processes. For instance, the form of your body is derived from countless atoms and molecules abiding by quantum processes. Are we to deny the relevance of quantum processes because they do not transpire at a macro level? Likewise, a constellation and summation of numerous micro quantum collapses of wave functions representing potentialities can provide for actualization of a given form at a macro level, with no formal collapse of a wave function occurring at the macro level. Much as atoms and molecules make up a macro form, this form can probably arise from micro-level quantum collapses of wave functions. Supporting the role of micro quantum processes in macro events, Hameroff and Penrose [31] propose that consciousness arises from orchestrated objective reduction activity (Orch OR), based on the notion of objective reduction (OR) of the quantum state.

Relating the quantum process described to future-present-past, the future only consists of potentialities represented by micro quantum wave functions. The present is where macro form occurs based on a constellation of micro quantum collapses of wave functions, derived from matter-energy interactions. So-called spontaneous collapse theories propose that a quantum system can collapse without measurement or observation, and this process becomes significant when the quantum system interacts with a macroscopic object [29] [30]. The actualized potentiality forms the past and is recorded or the information preserved at a quantum level, providing what I will refer to as a quantum actualization record. According to the Unitary Principle of quantum physics information is preserved [32], although Quantum Fields theory suggests that quantum fields might destroy information, at least at a particle level [33].

The process described is presented as three separate entities-future, present and past-but it almost certainly represents more of a continuous scenario derived from the interconnections. Hence, during a brief present potential form converts to actualized form derived from a constellation of micro quantum collapses of wave functions. The actualized form shifts to a quantum-based preservation of information (quantum actualization record) as the present shifts to the past. Rather than having abrupt points of demarcation it is a continuous process, likely contributing to the perception of time flowing. Linking this perspective to the general models of time presented earlier, it shares select features with each, although is very unique. Like the 4-D Block Model it maintains that past, present and future occur, but as active interconnected processes, instead of some static scenario. As with the Moving Spotlight Model the present is a narrow distinct 
zone, during which potentialities are actualized. The past grows from the present as with the Growing Block Model, but there is a future consisting of potentialities. Consistent with Presentism only the present transpires in the matter-energy world that we are familiar with, although the past and future do occur.

Critics might argue that the theory proposed appears to violate the notion, derived from special relativity, that objective time distinctions are not valid- time is relative to the observer. In response to this potential objection, we return to the issue of interconnected or entangled entities. Presence on Earth interconnects or entangles entities providing for essentially the same time, although the subjective experience of time almost certainly varies between species based on their unique perceptual capacities. If a person leaves the Earth and travels close to the speed of light-time dilation-that person will age much slower: The speed of light is in a sense the product of time and space, and if you travel through space near to the speed of light little is left for time, hence time slows. Aging more slowly means that time and hence the actualization of potentialities transpires at a slower rate for this person, than those still Earth bound. For example, cellular changes related to aging slow such that the person can live thousands or millions of years. When the "time traveler" returns to Earth he or she might only be a day or two older, but never younger, while people known to the person are now long deceased. The key point being that when entities become separated and are no longer connected or entangled, time can vary-it is relative. Hence special relativity is not violated by the proposed perspective on time.

Interesting, the universe seems to be structured to maintain the progressive actualization of potentialities, characterizing and perhaps defining time, because no object with mass can travel through space at the speed of light where time for the entity ceases, and it appears impossible to achieve absolute zero temperature, a scenario where all interactions, and hence collapses of wave functions, will stop. Furthermore, in the absence of any interaction entropy entails that matterenergy entities will progress from higher to lower order, ensuring that some potentiality is actualized [23].

An additional critique derived from the 4-D Block Model, is that future, past, and present just occur as a static entity. It is important to realize that a static perspective on time is largely derived from interpretations of certain equations [21] [25], and might not be accurate, given that equations often yield invalid outcomes [25]. Furthermore, even if valid it can be argued that the static future, present, and past did abide by the model proposed in this paper, and the 4-D Block Model just captures what transpired: The model is in essence a depiction of how in some dimension/s future potentialities became actualized forming the past, somewhat similar to how a documentary captures this progression. Conscious awareness might actually bias us to seeing the past and future as static, based upon how stable the present seems, even though it is actually quite fleeting and constantly changing even in the milliseconds to seconds range.

Casting doubt on the 4-D Block Model itself is how actual temporal sequences are evident in various phenomena, such as the progression of neural activity for 
events. Take the example of you turning a corner and encountering an attack dog. According to Ledoux's [34] fear circuit depiction sensory input travels to the thalamus, and then directly to the amygdala, producing a fear response in milliseconds. Occurring on a slower time scale, the information that reached the thalamus travels to the higher cortical regions, and based on the ensuing more sophisticated cognitive processing you realize that the dog is friendly and relax [34]. Perhaps the wagging tail and non-aggressive stance contribute to this revision, resulting in higher cortical suppression of the prior amygdala fear response. The passage of information from the thalamus to the amygdala, and the resultant conscious fear response, occurs prior to the passage of the information to the higher cortical regions, detailed processing, and suppression of the former amygdala response. There is a definite temporal sequence detectable at a neural level, with profound implications for evolutionary fitness. Hence, temporal sequences appear to be real consistent with the psychological arrow of time.

\section{Time-Based Evolution of Consciousness}

So far we have examined the nature of consciousness noting that awareness is the key feature, and time understood in terms of the future consisting of potentialities varying in probability, the present actualization of certain potentialities, and the past actualized potentialities in what I refer to as a quantum actualization record. However, we need to link consciousness to time. To do this we must know how my proposition regarding time relates to consciousness. A starting point is the present moment. Essentially, the present moment is where it all happens with potential occurrences being actualized. If you are too absorbed in your mobile device and step off the curb when a car is rapidly approaching, that fatal or very severe potential occurrence is actualized. Likewise, failure to react quickly to an attack dog will result in serious wounds. If an animal fails to respond rapidly and appropriately to a ripple on the surface of a watering hole, then that animal is a meal for the crocodile. The actualization of potentialities process occurs not at a macro level but derived from a constellation of numerous micro quantum wave function collapses.

Hence, what happens in the very brief present determines evolutionary fitness. Darwin [14] proposed natural selection stating that traits (behaviors included) that enhance survival and evolutionary fitness become more represented in succeeding generations. Given the tremendous significance of the present moment for evolutionary fitness, it is reasonable that a mechanism would evolve to facilitate rapid behavioral alterations in the present moment to minimize maladaptive potentialities from being actualized, and maximize the actualization of adaptive potentialities. It is proposed that this mechanism is consciousness providing an awareness of the present moment.

Awareness of the present moment provides the capacity, particularly in regards to motivation, for rapid alterations in behavior, minimizing the actualization of maladaptive outcomes and maximizing the actualization of adaptive outcomes. It might be suggested that unconsciousness can provide for this ca- 
pacity, eliminating the need for conscious awareness, at least in regards to adaptive alterations in behavior. However, while unconscious mental processing is very sophisticated, it cannot reliably motivate substantial alterations in behavior within a time frame of milliseconds to at most a few seconds. For instance, unconscious mental processing might detect a car approaching, the presence of an attack dog, or ripple on the surface of a watering hole and formulate a response, but unless the organism becomes consciously aware of the danger the time frame for behavioral alterations will pass. With conscious awareness a person is motivated to immediately halt their advance avoiding the oncoming car, react appropriately to the attack dog, and the animal jumps back depriving the lunging crocodile of a meal. In regards to positive circumstances, awareness of a potential partner showing interest can motivate you in the moment to make eye contact and smile, increasing the probability of a romance that might in turn lead to offspring. It all happens in the moment, and the motivation derived from conscious awareness is crucial for minimizing the actualization of maladaptive potentialities and maximizing adaptive outcomes.

Natural selection would then favor the evolution of consciousness as awareness of the present moment during which future potentialities are being actualized from matter-energy interactions. It is even possible that the apparent evolution of increasing cognitive sophistication is derived from the adaptive value of conscious awareness of the present moment. This process might help explain self-awareness in very cognitively advance species, because being self-aware adds to the adaptive value of conscious awareness for minimizing the actualization of maladaptive potentialities and maximizing the actualization of adaptive potentialities. For instance, if a person is aware that he tends to avoid interpersonal encounters, then when a potential partner makes eye contact and smiles, he can override his natural proclivity to avoid the situation and smile back. Selfawareness pertaining to physical limitations might lead a person to carry a walking stick when out for a stroll to defend against dangerous dogs. Selfawareness motivates behaviors, beyond what conscious awareness alone can facilitate, that enhance the actualization of adaptive outcomes and minimize the actualization of maladaptive outcomes.

Relevant to the model proposed is the time frame of consciousness and how it relates to the actualization of potentialities. The time frame for consciousness is in milliseconds to seconds [9] [35]. It takes about 300 to 500 milliseconds for a sensory input to achieve consciousness [9]. Conscious events have a duty cycle of about 100 milliseconds fading after a few seconds [35]. The time frame of consciousness then aligns very closely with the very short time frame during which potentialities are being actualized. This brief period of awareness provides for the richness of conscious experience covering sensory experiences, feelings, and thoughts, within the cognitive capacity of the given conscious organism. Hence, it should not be taken as a limited perspective on conscious experience. Instead the theory proposed provides a plausible reason for the evolution of consciousness with awareness of the present moment as the key aspect, and ac- 
counts for its apparent occurrence once a minimal level of cognitive sophistication has evolved.

\section{Implications for Time}

According to the theory presented, consciousness evolved in response to selection pressures associated with actualization of potentialities occurring in the present, to minimize the actualization of maladaptive potentialities and maximize the actualization of adaptive potentialities. This occurrence (if true) actually provides a logical proof for the presence of time distinctions in the realm of physics. The argument proceeds as follows: Natural selection is a real process being well validated scientifically. Consciousness has largely or fully evolved (natural selection) on the basis of time distinctions, providing awareness of potentialities being actualized in the present to optimize evolutionary fitness. Hence, time distinctions must be real, because if invalid they could not influence evolution.

\section{Summary}

According to the theory proposed here, consciousness has evolved based on time distinctions, and provides for an organism's awareness of potentialities being actualized in the present, based on matter-energy interactions, occurring in milliseconds and fading in seconds. Given the critical role of the actualization of potentialities process, and evolutionary significance of awareness of this process for rapid behavioral adjustments in the moment, the evolution of consciousness was highly probable, apparently arising with a basic level of cognitive sophistication. The evolution of consciousness based on time distinctions provides a logical proof of actual time distinctions.

\section{References}

[1] Linde, A. (1990) Particle Physics and Inflationary Cosmology. CRC Press, Boca Raton. https://doi.org/10.1201/b16971

[2] Smythies, J. (2003) Space, Time, and Consciousness, Journal of Consciousness Studies, 10, 47-56.

[3] Forstmann, M. and Burgmer, P. (2015) Adults Are Intuitive Mind-Body Dualists. Journal of Experimental Psychology: General, 144, 222-235. https://doi.org/10.1037/xge0000045

[4] Anglin, S.M. (2014) I Think, Therefore I Am? Examining Conceptions of the Self, Soul, and Mind. Consciousness \& Cognition, 29, 105-116. https://doi.org/10.1016/j.concog.2014.08.014

[5] Kumar, R. and Yeragani, V.K. (2010) Psyche and Soma: New Insights into the Connection. Indian Journal of Psychiatry, 52, S233-S239. https://doi.org/10.4103/0019-5545.69238

[6] Leitan, N.D. and Murray, G. (2014) The Mind-Body Relationship in Psychotherapy: Grounded Cognition as an Explanatory Framework. Frontiers in Psychology, 20 472-479. https://doi.org/10.3389/fpsyg.2014.00472

[7] Bonato, M., Zorzi, M. and Umilta, C. (2012) When Time Is Space: Evidence for a 
Mental Time Line. Neuroscience and Biobehavioral Reviews, 36, 2257-2273. https://doi.org/10.1016/j.neubiorev.2012.08.007

[8] Dietrich, A. (2003) Functional Neuroanatomy of Altered States of Consciousness: The Transient Hypofrontality Hypothesis. Consciousness and Cognition, 12, 231256. https://doi.org/10.1016/S1053-8100(02)00046-6

[9] Fabbro, F., Aglioti, S.M., Bergamasco, M., Clarici, A. and Panksepp, J. (2015) Evolutionary Aspects of Self and World Consciousness in Vertebrates. Frontiers in $\mathrm{Hu}$ man Neuroscience, 9, 157. https://doi.org/10.3389/fnhum.2015.00157

[10] Merker, B. (2007) Consciousness without a Cerebral Cortex: A Challenge for Neuroscience and Medicine. Behavioral and Brain Sciences, 30, 63-81. https://doi.org/10.1017/S0140525X07000891

[11] Alexander, I. and Cowey, A. (2010) Edges, Colour, and Awareness in Blindsight. Consciousness \& Cognition, 19, 520-533. https://doi.org/10.1016/j.concog.2010.01.008

[12] Tamietto, M., Cauda, F., Corazzini, L.I., Savazzi, S., Marzi, C.A., Goebel, R., Weiskrantz, L. and deGelder, B. (2010) Collicular Vision Guides Nonconscious Behavior. Journal of Cognitive Neuroscience, 22, 888-902. https://doi.org/10.1162/jocn.2009.21225

[13] Butler, A.B., Manger, P.R., Lindahl, B.I. and Arhem, P. (2005) Evolution of the Neural Basis of Consciousness: A Bird-Mammal Comparison. Bioessays, 27, 923936. https://doi.org/10.1002/bies.20280

[14] Darwin, C. (1858/1958) On the Origin of Species. Signet Classic, New York.

[15] Edelman, D.B. and Seth, A.K. (2009) Animal Consciousness: A Synthetic Approach. Trends in Neuroscience, 32, 476-484. https://doi.org/10.1016/j.tins.2009.05.008

[16] Mather, J.A. (2008) Cephalopod Consciousness: Behavioral Evidence. Consciousness and Cognition, 17, 37-48. https://doi.org/10.1016/j.concog.2006.11.006

[17] Cabanac, M., Cabanac, J. and Paren, A. (2009) The Emergence of Consciousness in Phyologeny. Behavioural Brain Research, 2, 267-272. https://doi.org/10.1016/j.bbr.2008.11.028

[18] Einstein, A. (1920) Relativity: The Special and General Theory. Henry Holt, New York.

[19] Callender, C. (2008) The Common Now. Philosophical Issues, 18, 339-361. https://doi.org/10.1111/j.1533-6077.2008.00151.x

[20] Dainton, B. (2010) Time, Passage, and Immediate Experience. In: Callender, C., Ed., The Oxford Handbook of Philosophy of Time, Oxford University Press, Oxford, 381-417.

[21] Barbour, J. (1999) The End of Time the Next Revolution in Physics. Oxford University Press, Oxford.

[22] Barrow, J.D. (2007) New Theories of Everything. Oxford University Press, Oxford.

[23] Deutsch, D. (1997) The Fabric of Reality. Penguin Books, London.

[24] Falk, D. (2008) In Search of Time: Journeys along a Curious Dimension. McClelland \& Stewart, Toronto.

[25] Lockwood, M. (2005) The Labyrinth of Time Introducing the Universe. Oxford University Press, Oxford.

[26] Maldacena, J. (2016) Black Holes, Wormholes, and the Secrets of Quantum Spacetime. Scientific American, 315, 26-31.

https://doi.org/10.1038/scientificamerican1116-26 
[27] Heisenberg, W. (1959) Physics and Philosophy: The Revolution in Modern Science. George Allen \& Unwin, London.

[28] Shankar, R. (1994) Principles of Quantum Mechanics. 2nd Edition, Springer, New York. https://doi.org/10.1007/978-1-4757-0576-8

[29] Mermin, N.D. (2012) Quantum Mechanics: Fixing the Shifty Split. Physics Today, 65, 8. https://doi.org/10.1063/PT.3.1618

[30] Von Baeyer, H.C. (2013) Quantum Weirdness? It's All in Your Head. Scientific American, 308, 46-51. https://doi.org/10.1038/scientificamerican0613-46

[31] Hameroff, S. and Penrose, R. (2014) Consciousness in the Universe: A Review of “Orch OR" Theory. Physics of Life Reviews, 11, 39-78. https://doi.org/10.1016/j.plrev.2013.08.002

[32] Braunstein, S.L., Pirandola, S. and Zyczkowski, K. (2013) Better Late than Never: Information Retrieval from Black Holes. Physical Review Letters, 110, Article ID: 101301. https://doi.org/10.1103/physrevlett.110.101301

[33] Weinberg, S. (2005) The Quantum Theory of Fields, Vol. 1: Foundations. Cambridge University Press, Cambridge.

[34] Ledoux, J. (1994) Cognitive-Emotional Interactions in the Brain: Nature of Emotions. Oxford University Press, Oxford.

[35] Baars, B.J. and Edelman, D.B. (2012) Consciousness, Biology, and Quantum Hypotheses. Physics of Life Reviews, 9, 285-294.

https://doi.org/10.1016/j.plrev.2012.07.001

\section{Scientific Research Publishing}

Submit or recommend next manuscript to SCIRP and we will provide best service for you:

Accepting pre-submission inquiries through Email, Facebook, LinkedIn, Twitter, etc.

A wide selection of journals (inclusive of 9 subjects, more than 200 journals)

Providing 24-hour high-quality service

User-friendly online submission system

Fair and swift peer-review system

Efficient typesetting and proofreading procedure

Display of the result of downloads and visits, as well as the number of cited articles

Maximum dissemination of your research work

Submit your manuscript at: http://papersubmission.scirp.org/

Or contact jbbs@scirp.org 